\title{
Opinión
}

\section{Evaluación de los exámenes preoperatorios}

\author{
Walter Rojas-Rivera
}

\section{Resumen}

Los exámenes preoperatorios son una práctica muy difundida en el quehacer del médico y el cirujano. Sin embargo, por lo general su uso no se basa en argumentos científicos o médicos.

La adecuada historia clínica y examen físico del paciente quirúrgico suelen ser suficientes para disminuir hasta en un $60 \%$ la cantidad de exámenes preoperatorios de rutina.

Implementando un método sistematizado, pero no inmodificable, la creación de guías de valoración preoperatoria permitirá al clínico tomar decisiones que le sugieran, en forma objetiva, solicitar exámenes con el fin de detectar o reafirmar los hallazgos de la anamnesis, para abandonar la tradicional "rutina preoperatoria".

Esta práctica consigue disminuir los costos de cada egreso, descongestionar los servicios de laboratorio y agilizar el tiempo preoperatorio del paciente.

Las guías son instrumentos dinámicos que se deben ajustar a las necesidades de cada población y adaptarse a los cambios en la tecnología y el conocimiento médico.

Descriptores: Exámenes preoperatorios, exámenes de rutina, valoración preoperatoria.

La valoración preoperatoria es una situación frecuente que el médico debe afrontar, muchas veces sin su correcto manejo y hasta de una manera intuitiva, que en el mejor de los casos se trata de una política institucional.

La evaluación preoperatoria es un proceso que trata de identificar la presencia de factores determinantes de riesgo perioperatorio, cuya detección y corrección permiten la disminución de la morbimortalidad ${ }^{4}$.

Los factores de riesgo pueden ser de 2 orígenes: aquellos propios del paciente y los que se asocian al tipo de cirugía que se planea realizar.

Los factores dependientes del paciente se relacionan con sus características, con la presencia de comorbilidad y con las alteraciones fisiológicas secundarias como resultado del cuadro que motiva la cirugía.

Walter Rojas-Rivera, MSc. Especialista en Anestesiología y Recuperación Hospital Dr.Tomas Casas Casajus. Caja Costarricense de Seguro Social.

Correo electrónico: walterrojasrivera@gmail.com

ISSN 0001-6002/2006/48/4/209-212 Acta Médica Costarricense, (2006 Colegio de Médicos y Cirujanos
Los factores de riesgo dependientes del procedimiento quirúrgico se relacionan con la repercusión funcional que determinará el procedimiento quirúrgico por realizar. Cada uno de estos factores debe ser evaluado en forma independiente, tratando de establecer las relaciones existentes entre ellos, para planificar las medidas que permitan disminuir su impacto. Este proceso se realizará fundamentalmente a través de la historia clínica y del examen físico.

Los llamados "exámenes de rutina" o "exámenes preoperatorios" debieran reemplazarse por el término "exámenes complementarios de los hallazgos de la historia o examen físico". Estos permitirán confirmar o cuantificar la presencia de un factor de riesgo, establecer el valor basal de algún parámetro que pueda ser modificado por la cirugía, cuya cuantificación preoperatoria pueda facilitar y optimizar el manejo del paciente ${ }^{1-4}$. 
La cirugía de urgencia se considera un factor de alto riesgo de morbilidad perioperatoria, atribuible a los problemas derivados de la falta de información de tiempo para realizar una adecuada evaluación clínica ${ }^{5}$.

Además, estos pacientes presentan alteraciones ausentes en los electivos, tales como estómago lleno o grados variables de déficit de volumen que, por razones de la patología quirúrgica o de la premura para realizar la cirugía, no alcanzan a ser corregidas adecuadamente. En este tipo de situación podría justificarse el uso más liberal de los exámenes de laboratorio, respaldados siempre por lo que el análisis de la situación clínica global de cada paciente hace aconsejable, y no en una batería preestablecida.

Esta claro pues, que la valoración preoperatoria no es un rubro que se pueda o se deba abordar como receta, no resulta conveniente desde el punto de vista financiero ni práctico.

Partiendo de estas premisas, grupos interdisciplinarios de especialistas, principalmente anestesiólogos, han elaborado protocolos para el manejo preoperatorio. Uno de los mejores ejemplos es el del National Institute for Clinical Excellence de Gran Bretaña, que en 2003 publicó “The use of routine preoperative tests for elective surgery" , que recapitula la experiencia en varios centros de salud británicos y genera una guía para la utilización de los exámenes preoperatorios. Sin embargo, por su extensión (más de 100 páginas) no se incluirá en este articulo.

Dicho análisis aborda múltiples aspectos: edad y género del enfermo, su estado según la American Society of Anestesiologyst (ASA), complejidad del procedimiento quirúrgico, apoyado en la historia clínica y el examen físico, pilares fundamentales en la adecuada valoración preoperatoria y puntos de partida para la correcta selección de los exámenes que el paciente requiere.

Desde el punto de vista estadístico, ya en la década de los 80, investigadores como Kaplan y cols, Turnbull y Buck $^{7}$, entre otros, plantearon análisis retrospectivos de la utilización de los exámenes de rutina, ambos con resultados muy similares, donde se demuestra que hasta un $60 \%$ de estos fueron innecesarios y que los resultados serían previstos con una buena historia clínica y examen físico.

Se puede, además, acotar que los métodos diagnósticos establecen límites normales con base en una curva de distribución o campana, en la que los valores que se alejan más de dos desviaciones estándar del nivel de confianza, se consideran anormales. Dado esto, el 5\% de los resultados de exámenes normales se considera anormal.

Al practicar 10 pruebas en un enfermo, surge una posibilidad del $40 \%$ de que al menos una de ellas sea anormal, no siéndolo de veras. Esto inevitablemente conduce a más pruebas y a tratamiento invasivos o no invasivos, que aumentan el riesgo de producir efectos perjudiciales al paciente.
Por otra parte, estudios como los de Billings ${ }^{3}$ y Roizen $^{5}$ mostraron que del $30 \%$ al $95 \%$ de los resultados inesperados obtenidos en las pruebas de rutina no fueron consignados en el expediente o no fueron analizados con detalle, de donde se deriva un hecho importante: la responsabilidad médico legal.

Respecto al tiempo de expiración de los estudios, es decir, el tiempo máximo que un estudio puede ser aceptado antes del acto quirúrgico, existen varios puntos de vista. Algunos autores consideran que 4 meses es un lapso adecuado, pero otros consideran que esto dependerá del tipo de estudio y de paciente; deben también tomarse en cuenta los cambios en la condición y medicación del paciente, ya que al repetir exámenes se incrementa la probabilidad de obtener resultados anormales en una persona normal.

La Pontificia Universidad Católica de Chile ha elaborado una guía general para la solicitud de exámenes para el preoperatorio (Cuadro 1). Debe aclararse que esta es una tabla que no implica patologías específicas o procedimientos quirúrgicos determinados; con el propósito de ampliar el tema y abarcar más posibilidades, se recomienda consultar el estudio del National Institute for Clinical Excellence de Gran Bretaña, disponible en la WEB ${ }^{6}$.

La guía resume en forma práctica muchos de los aspectos teóricos en los que los especialistas coinciden para realizar los distintos estudios, según la necesidad del paciente. Se han agregado algunas modificaciones con el objetivo de complementar esta tabla con el punto de vista de otros autores ${ }^{6-8}$.

Como complemento a lo planteado, se comentaron varios puntos relacionados con los diferentes estudios que usualmente se solicitan y se harán recomendaciones para cuando sea pertinente solicitarlas.

Con respecto al uroanálisis, nunca está indicado para el manejo anestésico; no obstante, es relevante para el cirujano en procedimientos como reemplazos protésicos.

La radiografía de tórax debe indicarse solo para obtener información clínica específica; es necesaria en cirugías particulares como la cardiotorácica y oncológica, es útil en pacientes con sintomatología cardiopulmonar, en las exacerbaciones recientes de los síntomas en pacientes con patología cardiopulmonar conocida, como: dolor torácico, disnea de esfuerzo, tos crónica, ortopnea, disnea paroxística nocturna, edema maleolar y disminución de la tolerancia al ejercicio, entre otros ${ }^{8}$.

Las pruebas de función hepática no se indican de rutina, solo en pacientes con historia reciente de enfermedad de ese órgano o del tracto biliar, por ejemplo.

Las pruebas hematológicas se deben utilizar en casos de sangrados anormales y célula enferma. El TP se indica en pacientes con leucemia o pacientes con disfunción hepática. 


\begin{tabular}{|c|c|c|c|c|c|c|c|c|c|c|c|c|}
\hline \multirow{2}{*}{\begin{tabular}{|c|} 
Condición preoperatoria \\
PACIENTES ASA \\
\end{tabular}} & \multicolumn{2}{|c|}{$\mathrm{Hto} / \mathrm{Hb}$} & \multirow{2}{*}{ 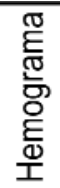 } & \multirow{2}{*}{\begin{tabular}{|l|} 
TP \\
TPT \\
\end{tabular}} & \multirow{2}{*}{\begin{tabular}{|l|} 
Rec \\
Plq \\
To Sgr
\end{tabular}} & \multirow{2}{*}{$\begin{array}{l}\frac{0}{2} \\
\frac{\pi}{0} \\
0\end{array}$} & \multirow{2}{*}{ 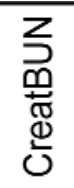 } & \multirow{2}{*}{$\begin{array}{l}\frac{\mathbb{\sigma}}{E} \\
\frac{0}{\Phi} \\
\frac{.0}{0}\end{array}$} & \multirow{2}{*}{ 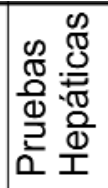 } & \multirow{2}{*}{ 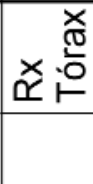 } & \multirow{2}{*}{ 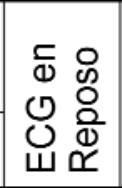 } & \multirow{2}{*}{. 든 } \\
\hline & $\mathrm{H}$ & M & & & & & & & & & & \\
\hline \multicolumn{13}{|l|}{$\begin{array}{l}\text { Procedimiento } \\
\text { quirúrgico }\end{array}$} \\
\hline \multicolumn{13}{|l|}{$\begin{array}{l}\text { Con pérdida de sangre } \\
>500 \mathrm{ml}\end{array}$} \\
\hline \multicolumn{13}{|l|}{$\begin{array}{l}\text { Con pérdida de sangre } \\
\text { de } 50-500 \mathrm{ml}\end{array}$} \\
\hline \multicolumn{13}{|l|}{$\begin{array}{l}\text { Sin pérdida de sangre } \\
<50 \mathrm{ml}\end{array}$} \\
\hline \multicolumn{13}{|l|}{ Edad } \\
\hline \multicolumn{13}{|l|}{$<40$ años } \\
\hline 40 - 49 años & & & & & & & & & & & Homb. & \\
\hline $50-64$ años & & & & & & & & & & & & Mujer \\
\hline 65 - 74 años & & & & & & & & & & & & Mujer \\
\hline$>74$ años & & & & & & & & & & & & Mujer \\
\hline \multicolumn{13}{|l|}{$\begin{array}{l}\text { Enfermedad } \\
\text { cardiovascular }\end{array}$} \\
\hline \multicolumn{13}{|l|}{ Enfermedad pulmonar } \\
\hline \multicolumn{13}{|l|}{ Cáncer } \\
\hline \multicolumn{13}{|l|}{ Radioterapia previa } \\
\hline \multicolumn{13}{|l|}{ Enfermedad hepática } \\
\hline \multicolumn{13}{|l|}{ Enfermedad biliar } \\
\hline \multicolumn{13}{|l|}{ Enfermedad Renal } \\
\hline \multicolumn{13}{|l|}{$\begin{array}{l}\text { Antecedente infección } \\
\text { urinaria }\end{array}$} \\
\hline \multicolumn{13}{|l|}{ Discrasia sanguínea } \\
\hline \multicolumn{13}{|l|}{ Diabetes Mellitus } \\
\hline $\begin{array}{l}\text { Fumador }>20 \\
\text { cigarrillos al día }\end{array}$ & & & & & & & & & & & & \\
\hline Enfermedad del SNC & & & & & & & & & & & & \\
\hline Uso de diuréticos & & & & & & & & & & & & \\
\hline Uso de digitálicos & & & & & & & & & & & & \\
\hline Uso de corticoides & & & & & & & & & & & & \\
\hline Uso de anticuagulantes & & & & & & & & & & & & \\
\hline Uso de inmunosupresores & & & & & & & & & & & & \\
\hline
\end{tabular}


Los estudios de química sanguínea como electrolitos, función renal, función hepática, proteínas y enzimas, se recomienda reservarlos para pacientes cuya historia clínica y examen físico sugieran alguna alteración.

El electrocardiograma está indicado según la edad, pero debe realizársele al diabético, considerando el estado, el tipo de cirugía, la condición del paciente y los beneficios que este estudio aportará a la disminución de la morbimortalidad.

La prueba de embarazo debe solicitarse a pacientes con historia de infertilidad, pacientes que se someterán a esterilización y pacientes con vida sexualmente activa que no utilizan ningún método de planificación. El hematocrito está indicado en mujeres en fase menstrual que serán sometidas a cirugía mayor o con metrorragia.

En los pacientes que reciben terapias de larga data, como por ejemplo, anticonvulsivantes o digoxina, es recomendable hacer la medición de los niveles sanguíneos de la droga.

Por su parte, la ASA concluye en sus publicaciones, que los exámenes de laboratorio o pruebas diagnósticas de rutina no son necesarios para la evaluación preanestesia de los pacientes. Se recomienda que cada centro elabore guías adaptadas a las necesidades de su población y que cada anestesiólogo ordene los exámenes cuando considere que los resultados que obtenga pueden influir en las decisiones, considerando los riesgos y el manejo de la anestesia y la cirugía ${ }^{1}$.

Los exámenes que intentan descubrir una enfermedad o desorden en un paciente asintomático, no contribuyen de manera importante en el proceso de valoración y manejo preoperatorio del paciente por parte del anestesiólogo².

\section{Conclusión}

Es recomendable establecer una guía institucional que permita la valoración eficiente y eficaz de los usuarios que serán sometidos a procedimientos quirúrgicos. Esta debe cubrir tanto los aspectos clínicos como los legales, y ser un respaldo para que el médico, luego de un exhaustivo estudio del paciente a través de la historia clínica y el examen físico, pueda definir de una forma más científica cuáles exámenes contribuirán con el adecuado manejo del paciente quirúrgico.

Al estudiar los costos se determina que parte importante del valor por la estancia operatoria se carga a servicios de apoyo, como Laboratorio y Rayos X, lo que lleva a su saturación el incremento en las posposiciones de cirugías, la realización de exámenes especiales innecesarios, e inclusive la posibilidad de incurrir en iatrogenia.
Finalmente, es preciso resaltar que las guías son un instrumento dinámico para orientar al médico, no un patrón inamovible del cual no puede salirse o que puede cambiarlo con el tiempo; existen guías aplicadas a diferentes tipos de pacientes intervenciones quirúrgicas, pero ante todo, una buena exploración del paciente ofrecerá más información que los “exámenes preoperatorios de rutina”.

\section{Referencias}

American Society of Anestesiologysts.[homepage on the internet].New York: c2002. [update 2002 Jan 03; cited 2006 Jan 20].Statement on routine preoperative laboratory and diagnostic screening. Disponible en: www.asahq.org/Standards/28.html.1993.

2. American Society of Anestesiologysts. Practice advisory for preanesthesia evaluation: a report by the American Society of Anestesiologysts. Task force on preanesthesia evaluation. Anesthesiology 2002:96, 485-96.

3. Billings PJ, Richards R, Davies JP, Audrey DA: An audit of preoperative investigation of surgical patients. Ann R Coll Surg Engl 75:205,93.

4. Concha-Pinto M, Merts-Kaizer V, González-Arellano A. Evaluación preoperatoria en pediatría. Sociedad de Anestesiología de Chile. [serial on the internet]. 2002 Feb.[ cited 2006 Jan 20]; 31(1): [about 8 p]. Disponible en: www.socanestesia.cl/index.asp

5. Roizen MF. Evaluación preoperatoria. En Miller RD (ed): Anestesia, ed 2. Barcelona, Ediciones Doyma, p 675-698.

6. The National Institute for Clinical Excellence (NICE). [ homepage on the internet]. London: NHS; c2003 [update 2003 jun; cited 2006 Jan 20]. Guidelines for routine preoperative testing. Disponible en http://www.nice.org.uk

7. Turnbull JM, Buck C: The value of preoperative screening investigations in otherwise healthy individuals. Arch Intern Med 147:1101, 1987.

8. University Hospital of Cleveland. Department of Anesthesiology. .[homepage on the internet]. Ohio: c2006 [update 2002 Aug; cited 2006 Jan 12]. Tables summarising suggested preoperative laboratory testing. Adult Preoperative Testing Guidelines.Disponible en:www.uhcanesthesia.com/PAT/Pat-staff/lat-guidelines/pattesting/view 